\title{
Personas con discapacidad: barreras de acceso al empleo y algunas alternativas de inclusión en Colombia*
}

\author{
People with disabilities: barriers to access to employment \\ and some alternatives of inclusion in Colombia \\ Natalia Eugenia Gómez Rúa ${ }^{1 \otimes}$, Ana Lucía Pérez Medina ${ }^{2} \mathbf{1}$ \\ Diana María López Arango ${ }^{3 凶}$, María Fernanda Medina Ruiz ${ }^{4}{ }^{\prime}$
}

Fecha correspondencia:

Recibido: 14 de agosto de 2020.

Revisión: 13 de octubre de 2020.

Aceptado: 27 de octubre de 2020

Forma de citar:

Gómez, Natalia Eugenia; Pérez, Ana

Lucía; López, Diana María; Medina,

María Fernanda. "Personas con

discapacidad: barreras de acceso al

empleo y algunas alternativas de

inclusión en Colombia." En: Revista

CES Derecho. Vol. 11, No. 2, junio a

diciembre de 2020, 3-24.

Open access

Términos de uso

Licencia creative commons

Ética de publicaciones

Revisión por pares

Gestión por Open Journal System

DOl: http://dx.doi.org/10.21615/

cesder.11.2.1

ISSN: 2145-7719

Sobre el artículo:

Producto de los proyectos de investigación "El desarrollo de capacidades de las personas con discapacidad: una mirada desde enfoque de derechos de la salud laboral" de los grupos de investigación Observatorio de la Salud Pública y Estudios Jurídicos de la Universidad CES y "Barreras

\section{Resumen}

El objetivo de este artículo es identificar las alternativas para minimizar las barreras de empleo de las personas con discapacidad en Colombia a partir del derecho al trabajo digno. Se revisa la producción normativa sobre este eje temático con posterioridad a la ratificación de la Convención de los Derechos de las Personas con Discapacidad (Organización de Naciones Unidas, 2006), es decir, desde el año 2011, las barreras identificadas para el acceso al empleo; y posteriormente, se presentan las alternativas planteadas en el país para acceso al trabajo de esta población de orden estatal y privada. La investigación es cualitativa y su enfoque metodológico es el hermenéutico, el cual se realizó a través de un análisis de contenido de normas y literatura sobre el tema objeto de estudio. Los resultados evidenciaron que el desarrollo normativo es amplio y suficiente para garantizar el bienestar en los entornos laborales. Asimismo, se identifican desde las leyes y diferentes autores las barreras que tiene esta población para la participación y la igualdad en el trabajo; evidenciando, a su vez, la existencia de alternativas que han contribuido a minimizar esas barreras y las cuales han aportado para la inclusión social; no obstante, pueden tornarse insuficientes respeto a las tasas de desempleo, las ofertas laborales y los perfiles ocupaciones que se ofrecen siendo necesario crear puentes más sólidos de carácter educativo y empresarial que no solamente permitan el acceso al empleo sino a condiciones de permanencia y seguimiento en materia laboral a las personas con discapacidad.

Palabras claves: Barreras, Empleo, Normativa, Personas con discapacidad, Trabajo.

\section{Abstract}

The objective of this article is to identify alternatives to minimize employment barriers for people with disabilities in Colombia based on the right to decent work. The normative production on this thematic axis is reviewed after the ratification of the Convention on the Rights of Persons with Disabilities (United Nations Organization, 2006), that is, since 2011, the barriers identified for access to employment and subsequently the alternatives 
que limitan en derecho

al trabajo digno de la

población en situación de

discapacidad" adscrito al

Grupo de Estudios Jurídicos

de la Facultad de Derecho.

Sobre los autores:

1. Docente Universidad CES

2. Estudiante Maestría

Universidad CES.

3. Estudiante Maestría

Universidad CES.

4. Estudiante de Pregrado

Universidad CES. proposed in the country for access to the work of this state and private population are presented. The research is qualitative and its methodological approach is the hermeneutic and was carried out through an analysis of the content of standards and literature on the subject under study. The results showed that regulatory development is broad and sufficient to guarantee well-being in work environments. Likewise, the barriers that this population has for participation and equality at work are identified from the laws and different authors and in turn there are alternatives that have contributed to minimize those barriers which have contributed to social inclusion but may become insufficient respect for Unemployment rates, job offers and occupational profiles that are offered being necessary to create stronger educational and business bridges that not only allow access to employment but also conditions of permanence and follow-up in labor matters for people with disabilities.

Keywords: Barriers, Employment, People with disabilities, Regulations, Work.

\section{Introducción}

La ratificación de la Convención de los Derechos de las Personas con Discapacidad (Organización de Naciones Unidas, 2006) por parte del estado colombiano, implica el deber de incluir en las propuestas legislativas el concepto acogido de discapacidad en este instrumento internacional, es decir, el resultado de la interacción tanto entre las deficiencias humanas como en el medio en el que se desenvuelve y no sólo una condición de salud propia de la persona. En este sentido, en Colombia, el artículo 2, numeral 1 de la Ley 1618, define las personas con discapacidad como "aquellas que tengan deficiencias físicas, mentales, intelectuales o sensoriales a mediano y a largo plazo que, al interactuar con diversas barreras incluyendo las actitudinales, pueden impedir su participación plena y efectiva en la sociedad, en igualdad de condiciones con las demás". Adicionalmente identifica, de una manera expresa, las barreras a las que se debe enfrentar la población que sufre algún tipo de discapacidad y las clasifica en actitudinales, sociales, culturales, físicas, arquitectónicas y de comunicación (Congreso de la República de Colombia, 2013a).

Con relación a las condiciones de empleo, en el Informe Mundial sobre la Discapacidad, se destaca que a partir de diferentes estudios en países tanto emergentes como desarrollados, las tasas de empleo de las personas con discapacidad respecto a las personas sin discapacidad son considerablemente menores y las de desempleo muchísimo más altas (Organización Mundial de la Salud \& Banco Mundial, 2011). También se argumenta que las bajas tasas de participación en el mercado laboral constituyen una de las razones importantes por las cuales la discapacidad puede conducir a la pobreza. Por su parte, la Organización Internacional del Trabajo (OIT), afirma que "de los mil millones de personas con discapacidad en el mundo, al menos 785 millones están en edad de trabajar" (Organización Internacional del Trabajo, 2015). Al respecto, la Comisión Económica para América Latina y el Caribe (CEPAL) indica que "constituyen un grupo especialmente vulnerable, puesto que la desigualdad que los afecta parte de su difícil acceso al mundo del trabajo, esfera definitoria de la persona en el sistema capitalista, que mediante un proceso de construcción política ha ligado la esencia humana al trabajo" (Stang, 2011).

En este sentido, la Organización Internacional del Trabajo (OIT) ha indicado que "tiene un compromiso de larga duración para promover la justicia social y lograr trabajo decente para las personas con discapacidad" («Discapacidad y trabajo», 2016). Para estos fines la mencionada organización trazó una estrategia y plan de Acción para la inclusión de la discapacidad (Organización Internacional del Trabajo, 2015), el cual 
incluye la promoción del empleo y subraya el hecho de que muchos países no brindan la suficiente cobertura a las personas con discapacidad en sus programas de protección social y que, cuando se han adoptado disposiciones a tal efecto, éstas no suelen facilitar su participación en el mercado laboral (International Labour Organization, 2014).

En Colombia, al año 2018, la Sala Situacional de las Personas con Discapacidad del Ministerio de Salud y Protección Social, reporta que en el Registro para la Localización y Caracterización de Personas con Discapacidad (RLCPD) aparecen 1.418.065 ciudadanos inscritos; además se afirma que por cada 100.000 habitantes hay 2.818 personas con discapacidad registradas (Ministerio de Salud y Protección Social, 2018). Concretamente respecto al acceso efectivo al trabajo de las personas con discapacidad, el Ministerio de Trabajo indica que a corte del 2014, a través de la Unidad del Servicio Público de Empleo, se inscribieron a 3.526 personas con alguna discapacidad y se vincularon cerca de 1.500 empresarios y actores sociales en los 42 encuentros de 'Oportunidades Incluyentes' para la vinculación laboral realizados en 29 departamentos (Ministerio de Salud y Protección Social, 2015). No obstante lo anterior, autores como Moreno et al, afirman que el Departamento Administrativo Nacional de Estadística (DANE) estima que apenas el 7\% de la población colombiana con discapacidad accede a un trabajo, lo cual impide, a su vez, permanencia y condiciones dignas de trabajo (Moreno, Cortés, Cárdenas, Giraldo, \& Mena, 2013).

En la triada de trabajo, empleo y discapacidad, debe tenerse en cuenta que el trabajo es un componente básico del bienestar de las personas. De acuerdo con Benavides et al, se refiere al "esfuerzo físico y mental que realizamos para satisfacer una necesidad, a todo tipo de trabajo, incluido el trabajo forzado (esclavo) o infantil, y debemos incluir el trabajo doméstico o de un estudiante". (García, Delclós, \& Benavides, Fernando, 2013). De dicho concepto se derivan la ocupación y el empleo. Respecto a la ocupación señalan los mismos autores que se trata del "trabajo realizado a cambio de algún tipo de remuneración, con o sin relación formal, incluye el trabajo informal" (García et al., 2013). Correspondiente al empleo indican que es "trabajo remunerado realizado bajo algún tipo de relación legal, sea como asalariado con contrato temporal o permanente, o como autónomo" (García et al., 2013).

En este artículo, la categoría de análisis es concretamente el empleo, que en coherencia con lo anterior también ha sido definido por la Organización Internacional del Trabajo (OIT) como "trabajo efectuado a cambio de pago (salario, sueldo, comisiones, propinas, pagos a destajo o pagos en especie) sin importar la relación de dependencia (si es empleo dependiente-asalariado, o independiente-autoempleo)" (Organización Internacional del Trabajo, 2004). Es un término estrechamente vinculado al Trabajo Decente; el cual "busca expresar lo que debería ser, en el mundo globalizado, un buen trabajo o un empleo digno. El trabajo que dignifica y permite el desarrollo de las propias capacidades no es cualquier trabajo; no es decente el trabajo que se realiza sin respeto a los principios y derechos laborales fundamentales, ni el que no permite un ingreso justo y proporcional al esfuerzo realizado, sin discriminación de género o de cualquier otro tipo, ni el que se lleva a cabo sin protección social, ni aquel que excluye el diálogo social" (Organización Internacional del Trabajo, 2004).

En el contexto internacional, hay dos enfoques generales para proporcionar acceso en el lugar de trabajo. El enfoque de América del Norte, desarrollado en los Estados Unidos y Canadá, en el que los empleadores están obligados a acomodar las limitaciones conocidas de los empleados con discapacidad; y el enfoque europeo, en el 
que los empleadores están obligados a hacer la totalidad de sus lugares de trabajo accesible (Metts, 2000).

De acuerdo con lo anterior, en América Latina, el enfoque es facultativo; basado más en la motivación y en los incentivos para los empleadores. Al respecto, la Organización Iberoamericana de la Seguridad Social (OISS), señala que se han trazado estrategias para promover el empleo de las personas con discapacidad; como cuotas obligatorias de reserva de puestos de trabajo en la administración pública y en la empresa privada, preferencia a empresas que contraten personas con discapacidad en la adquisición de bienes o en la contratación de servicios, servicios públicos de empleo y orientación laboral, incentivos a las empresas privadas para que contraten personas con discapacidad, medidas adicionales para facilitar el trabajo, acciones para sensibilizar a las empresas sobre el empleo de las personas con discapacidad, formación ocupacional, empleo con apoyo, entre otras (Palacios, 2008).

Sin embargo, en Iberoamérica los obstáculos a los que se enfrentan las personas con discapacidad para acceder a un empleo siguen siendo numerosos. Problemas para acceder a la educación, falta de accesibilidad de los transportes, del entorno construido y de la información, prejuicios acerca de la discapacidad, etc. Todo ello origina que aproximadamente el $80 \%$ de las personas con discapacidad se encuentren desempleada en algunas zonas de la región; lo cual perpetúa el círculo vicioso entre pobreza y discapacidad. (Organización Iberoamericana de Seguridad Social, 2014).

Sobre el acceso al empleo en el país, la Coalición Colombiana por la Implementación de la Convención sobre los Derechos de las Personas con Discapacidad (Organización de Naciones Unidas, 2006), en el marco de los denominados informes de sombra sobre el cumplimiento de la misma, señala que por medio de la Ley 361 de 1997 se pretendió evitar la discriminación por motivos discapacidad, a través de la figura de estabilidad laboral reforzada; pero la interpretación de esta norma por parte de la Corte Constitucional, al extender la protección a toda persona que presente una afectación en su estado de salud sea de manera temporal o permanente, constituye hoy en día una de las principales barreras de acceso al mercado laboral para personas con discapacidad.

Lo anterior, debido a que "los empleadores ven en la contratación de personas con discapacidad, una normativa de protección laboral absoluta que hace imposible la terminación de sus contratos, aun cuando medie causales de justificación, por lo que los asesores laboralistas desaconsejan la contratación de esta población, aunque existen incentivos tributarios para su contratación" (Organización de Naciones Unidas, 2006, p. 41).

Por el contrario, de la premisa de discriminación laboral atribuida a los empleadores, no es pertinente afirmar que no hay acciones encaminadas a buscar el derecho al trabajo digno de las personas con discapacidad a través de alternativas para minimizar las barreras de empleo como ligeramente puede interpretarse en nuestro entorno; ya que, tanto a nivel internacional como nacional, se han emprendido acciones para su contratación y se han compartido experiencias exitosas tanto de empresas del sector público como privado (Farné, 2003; Pacto de Productividad. Promoviendo la Inclusión Laboral de las personas con discapacidad, 2015; Stang, 2011; Véliz \& Espinoza, 2010). 
En aras de revisar de forma crítica el tema en el contexto colombiano, se expone en la primera parte el desarrollo normativo relacionadas con el derecho al trabajo digno de las personas con discapacidad; posteriormente, en la segunda parte, se describen las barreras con mayor visibilidad respecto a su goce; por último, en la tercera parte, se analizan las principales estrategias implementadas en el país para minimizar las barreras de empleo de esta población.

\section{Normativa relacionada con el derecho al trabajo digno de las personas en situación de discapacidad}

En la legislación colombiana se han acogido diferentes normas internacionales relacionadas con el acceso al empleo de las Personas con Discapacidad, adicionales a la citada Convención, de las cuales sobresalen las siguientes: Convenio 159 de la OIT sobre readaptación profesional y empleo (Organización Internacional del Trabajo, 1983), las Normas Uniformes sobre la Igualdad de Oportunidades para las personas con discapacidad de la ONU (Organización Internacional del Trabajo, 1996), y la Convención interamericana para la eliminación de todas las formas de discriminación contra las personas con discapacidad de la ONU (Organización de Estados Americanos, 1999).

Por su parte, la Constitución Política, advierte la obligación de adelantar una política de previsión, rehabilitación e integración social para los disminuidos físicos, sensoriales y psíquico e impone al Estado el deber de propiciar la ubicación laboral de las personas en edad de trabajar, así como de garantizar a los minusválidos el derecho a un trabajo acorde con sus condiciones de salud (Asamblea Nacional Constituyente, 1991). Los términos disminuidos y minusválidos, hoy a la luz de la Convención de los Derechos de las Personas con Discapacidad deben entenderse como personas con discapacidad y en este sentido lo indicó el Alto Tribunal en la sentencia C-425 (Corte Constitucional, 2005).

Estos postulados han sido puestos en práctica por los órganos delegados de aprobar la normativa en Colombia. Respecto al derecho al trabajo digno de las personas con discapacidad, las principales normas que se han proferido en Colombia son las siguientes. Tabla 1.

Tabla 1. Normas relacionadas con el derecho al trabajo digno de las personas con discapacidad

\section{NORMA DERECHO AL TRABAJO DIGNO DE LAS PERSONAS EN SITUACIÓN DE DISCAPACIDAD.}

Ley 82 de 1989 Ratifica el Convenio sobre la readaptación profesional y el empleo de personas con discapacidad. Tiene como premisa la obtención, conservación y progreso del empleo.

Se establece la obligación del gobierno de desarrollar programas especiales de gestión de empleo para personas $\begin{array}{ll}\text { Decreto } 2177 & \text { con discapacidad, rehabilitadas integral y profesionalmente y en condiciones de competitividad laboral, mediante el } \\ \text { de } \mathbf{1 9 8 9} & \text { impulso de actividades y el diseño de mecanismos tendientes a estimular la creación de diversas formas de trabajo }\end{array}$ de 1989 dependiente.

Decreto 970 de

Promulga el convenio sobre la readaptación profesional y el empleo ratificado en la Ley 82 de 1989.

Ley 191 de Se dictan disposiciones sobre Zonas de Frontera y estipula que todas las empresas beneficiadas por esta ley 1995 deberán tener en cuenta en su vinculación laboral a las personas con discapacidad residentes en dichas zonas.

Ley 443 de 1998
Promueve la adopción de medidas tendientes a garantizar en igualdad de oportunidades las condiciones de acceso al servicio público, en empleos de carrera administrativa, con el fin de proporcionarles un trabajo acorde con sus condiciones de salud. 


\section{NORMA DERECHO AL TRABAJO DIGNO DE LAS PERSONAS EN SITUACIÓN DE DISCAPACIDAD.} trabajadores adicionales a los que tenían en promedio en el año 2002, con una disminución de su capacidad laboral superior al veinticinco por ciento (25\%) debidamente calificada por la entidad competente. Igualmente, garantiza a las personas empleadas que tengan a su cargo hijos con discapacidad, o capacidad física disminuida, doble cuota del subsidio sin limitación a su edad.

Ley 762 de Incluye medidas para eliminar todas las formas de discriminación en el empleo.
$2002 \quad$.

Decreto 205 de Proponer y promover la ejecución de políticas de readaptación profesional y generación de empleo para personas 2003 con discapacidad Decreto 827 de Se reglamenta la administración y gestión del Fondo para el Fomento del Empleo y Protección al Desempleo

Ley 982 de 2005

Ley $361 \mathrm{de}$ 1997

Ley $1147 \mathrm{de}$ 2007
Establece un régimen especial de protección y promoción laboral para las personas sordas y ciegas.

Prevé la adopción de medidas que permitan equiparar oportunidades para las personas con discapacidad en el ámbito laboral.

El Estado se compromete a la formulación de políticas públicas para asegurar el Derecho al Trabajo con equiparación de oportunidades, creando el Consejo Nacional de Discapacidad, cuya función según el artículo 8 , num.2 es el de "organismo consultor, asesor institucional y de verificación, seguimiento y evaluación del Sistema y de la Política Pública Nacional de Discapacidad
Ley 1221 de 2008

Ley 1306 de 2009

Ley 1429 de 2010

Decreto 4910

de 2011

Decreto 884 de 2012

Ley 1618 de 2013

Decreto 2011 de 2017

Promover y regular el Teletrabajo; así como la implementación de una política para personas con discapacidad.

Normas para la Protección de Personas con Discapacidad Mental y su derecho al empleo.

Ley de formalización y generación del empleo. Se establecen beneficios para los empleadores que contraten a personas en condición de discapacidad. los nuevos empleos que correspondan a las personas, entre otras, con discapacidad. promover la inclusión laboral de población con discapacidad mediante el teletrabajo, a través del acceso a las tecnologías de la información y las comunicaciones para el contacto entre el trabajador y la empresa.

Garantizar el derecho al trabajo de las personas con discapacidad. Fomenta el empleo de las personas con discapacidad y el derecho al trabajo en términos de igualdad de oportunidades, equidad e inclusión. Así mismo, beneficios de preferencias en los procesos de adjudicación y celebración de contratos con el Estado y el discapacidad. Único del Sector de Función Pública, en lo relacionado con el porcentaje de vinculación laboral de personas con
Reglamenta la Ley 1429 de 2010. Concretamente, en lo referente a beneficios tributarios y parafiscales, respecto de

Reglamenta la Ley 1228 de 2008. Donde el Ministerio de Tecnologías de la Información y las Comunicaciones, debe otorgamiento de créditos o subvenciones de organismos estatales, para empresas que contraten a personas con

Por el cual se adiciona el Capítulo 2 al Título 12 de la Parte 2 del Libro 2 del Decreto 1083 de 2015, Reglamentario discapacidad en el sector público

Por el cual se integra el Consejo para la Inclusión de la Discapacidad articulado al Sistema Nacional de

Decreto 2177 de 2017 Discapacidad, cuyo objeto será coordinar las acciones que el sector privado adelante para coadyuvar al ejercicio de los derechos y la inclusión social, laboral y productiva de las personas con discapacidad, sus familias y cuidadores.

Decreto 392 de Por el cual se reglamentan los numerales 1, y 8 del artículo 13 de la Ley 1618 de 2013, sobre incentivos en 2018 Procesos de Contratación en favor de personas con discapacidad.

Ley 1955 de 2019
Plan Nacional de Desarrollo 2018-2022. Uno de los pactos del plan es la inclusión de las personas con discapacidad en materia de empleo; así mismo promover acciones afirmativas como la reubicación laboral o ser los últimos retirados. 
De las 22 normas proferidas puede concluirse que 20 han sido proferidas con posterioridad a la Constitución Política (Asamblea Nacional Constituyente, 1991), tendientes a garantizar los principios y derechos laborales fundamentales de las personas con discapacidad en el mundo del trabajo. Como puede observarse en la Tabla 1 , principalmente se ha tratado de incentivar la inclusión laboral creando beneficios tributarios para empleadores que vinculen a las personas con discapacidad. Lo anterior se puede observar en la Ley 789 de 2002 (Congreso de la República de Colombia, 2002, p. 789), la Ley 1429 de 2010 (Congreso de la República de, 2010).

Por otra parte, sobre la protección en el trabajo, una norma fundamental es la Ley 361 de 1997, por medio de la cual se establecen mecanismos de integración social de las personas con limitación y establece obligaciones al empleador y al Estado. Concretamente el artículo 26 de la Ley 361 de 1997 (Congreso de la República de Colombia, 1997, p. 361), el cual trata de medidas para que las personas con discapacidad no sean despedidas debido a su limitación física, psíquica o sensorial y en caso de que sea imposible reubicarlas, el empleador debe solicitar el permiso ante la autoridad administrativa competente para que se garanticen los derechos de ese trabajador. En caso de omitir el trámite, además de las prestaciones sociales de ley adeudadas al trabajador, este puede ejercer acciones legales para que se cancele una indemnización equivalente a 180 días de salario e incluso buscar ser reintegrado. De este presupuesto y algunas normas constitucionales se ha derivado la denominada estabilidad ocupacional reforzada (Corte Constitucional, 2018)

Adicionalmente, puede afirmarse que la apuesta regulatoria se consolida en la Ley 1618 de 2013, la cual es un hito por ser una norma estatutaria de rango superior con carácter prioritario para su implementación (Congreso de la República de Colombia, 2013a); y sus decretos reglamentarios, que si bien aún no cuenta con evaluación por parte de organismos estatales y/o de la ciudadanía se pueden estimar como relevantes para lograr la protección del derecho al trabajo digno de las personas con discapacidad.

Específicamente sobre el Decreto 2011 de 2017 (Ministerio de Trabajo, 2017a), aunque la obligatoriedad de porcentaje de vinculación laboral de personas con discapacidad es únicamente para las entidades del sector públicas, su aplicación dejará una ruta para que se promuevan normas en el sector privado y no se quede la citada Ley en simplemente una aspiración de nuestro ordenamiento jurídico. Una crítica a los preceptos del Decreto 2011 es que prevé una ejecución progresiva hasta el 2027 de acuerdo con el número de trabajadores de la entidad pública, y por lo tanto su eficacia se diluye en el tiempo hasta que sea una realidad en todas las empresas del Estado.

En esta misma línea, el Decreto 2177 de 2017, por el cual se integró el Consejo para la Inclusión de la discapacidad en articulación con el Sistema Nacional de Discapacidad por parte del Ministerio de Trabajo, si bien ha cumplido un papel relevante en los escenarios políticos, aún queda pendiente su tarea de "la creación de la red nacional de empresas incluyentes, así como mecanismos de certificación y reconocimiento para las empresas que incluyan a personas en situación de discapacidad" (Ministerio de Trabajo, 2017b)

Por último, el Departamento Nacional de Planeación expidió el Decreto 392, que reglamenta los numerales 1 y 8 del artículo 13 de la Ley 1618 de 2013, relacionados con los incentivos en procesos de contratación a favor de personas en situación de 
discapacidad; de esta manera, en licitaciones públicas y concursos de méritos, se incentiva el sistema de preferencias en razón de las personas con discapacidad; las entidades estatales deberán otorgar el $1 \%$ del total de los puntos establecidos en el pliego de condiciones, a los proponentes que acrediten la vinculación de trabajadores con discapacidad en su planta de personal. (Ministerio de Hacienda y Crédito Público, 2018, p. 392). Al respecto, si bien el propósito puede ser positivo, el porcentaje establecido obstaculiza una verdadera inclusión al no ser significativo con relación a los cargos de carácter público que ofrece el Estado en el país.

En consonancia con las normas, las políticas públicas en la región también son importantes. Al respecto, el gobierno expidió el CONPES 166 de 2013; dentro del cual se reconoce la evolución del término discapacidad, el interés por la inclusión laboral y el reconocimiento de los factores relacionados con el trabajo. Se debe agregar que en el texto se considera importante desarrollar capacidades humanas y acciones para fortalecer el papel de los empleadores y las familias, en aras a que las personas con discapacidad puedan afrontar las barreras para la igualdad de condiciones y participación (Consejo Nacional de Política Económica y Social, 2013).

De acuerdo con la Política Nacional de Discapacidad, en Medellín se promulgó el Acuerdo Municipal No 086 de 2006 (Alcaldía de Medellín, 2009) con el propósito de adoptar la citada política en la ciudad; el cual fue reglamentado a través del Decreto 221 de 2011 de la Alcaldía de Medellín. Sobre programas para el empleo, es reconocido en la ciudad el trabajo realizado por la Unidad de Discapacidad adscrita a la Secretaría de Inclusión Social, familia y derechos humanos, a través de su proyecto Ser Capaz, en la línea denominada Inclusión Sociolaboral que "ofrece las etapas de evaluación, orientación, formación, adaptación psicosocial y laboral y promoción para el empleo, a personas con discapacidad entre los 18 y 59 años, de acuerdo a su perfil y a las opciones laborales del medio." (Secretaría de Inclusión Social, Familia y Derechos Humanos., 2019).

Es importante resaltar que para la salud pública, las problemáticas que enfrentan las personas en situación de discapacidad, también ha sido relevante el Plan Decenal de Salud Pública 2012-2021, el cual se ha fijado especialmente en la dimensión ambiental que debe protegerse como parte de la población en situación de vulnerabilidad (Ministerio de Salud y Protección Social, 2013); siendo en ámbito laboral de gran significación para identificar las barreras que presentan, especialmente para el acceso al trabajo digno como se explica a continuación.

En articulación con lo anterior, el Plan Nacional de desarrollo (PND) 2018-2022 pretende realizar un pacto para avanzar en la igualdad de oportunidades, inclusión social y productiva de las personas en situación de discapacidad; removiendo las barreras con coordinación y acciones intersectoriales decididas. Además, se plantea como objetivos actualizar e implementar las acciones de la Política Pública Nacional de Discapacidad e Inclusión Social, para eliminar toda forma de discriminación; implementar programas de educación inclusiva orientados a una efectiva inclusión social y productiva de las personas en situación de discapacidad; mecanismos de inclusión productiva para la generación de ingresos y la seguridad económica de las personas en situación de discapacidad; crear un Plan Nacional de Accesibilidad que asegure la participación de las personas en situación de discapacidad en igualdad de condiciones en el entorno físico, el transporte, la información y las comunicaciones, incluidas las TIC, tanto en zonas urbanas como rurales; adoptar las medidas para la garantía del pleno reconocimiento de los derechos y para el ejercicio de la 
capacidad legal de las personas en situación de discapacidad y el acceso a la justicia; fortalecer la oferta existente, especialmente en los programas de asistencia y protección social y articularla en el marco del cuidado que define el PND en el pacto de equidad para las mujeres. (Departamento Nacional de Planeación, 2018)

En conclusión, los referentes legales explicados anteriormente son un avance importante para el acceso al empleo y la concientización de las empresas de tener una política inclusiva. No obstante, sigue ausente un marco regulador para los buenos propósitos de accesibilidad laboral, especialmente de lo propuesto en la Ley 1618 de 2013. Por lo anterior, se requiere ir más allá de un compromiso social y plantear obligaciones claras para las empresas y garantías para empleadores y trabajadores que participen activamente en la minimización de barreras para la participación y la igualdad de condiciones. Conocer las barreras más visibles, podría contribuir a las propuestas reglamentarias necesarias para la efectividad de los derechos de esta población en el campo laboral.

\section{Barreras para el acceso al trabajo digno de las personas en situación de discapacidad}

En el en marco del trabajo decente, el acceso a un empleo digno debe ser entendido como "la posibilidad de conseguir un trabajo de calidad, digno y justo que genera condiciones de cohesión social y contribuye a la determinación de sociedades más saludables" (Benach \& Muntaner, 2010). Por lo que se refiere a que se tienen en cuenta cuestiones como las condiciones de salario, contratos de trabajo y trato justo, entre otros (Benavídez, Boix, Rodrigo, \& Gil, 2013). Específicamente, con relación a las personas con discapacidad, se ha precisado que la inclusión laboral debe darse dentro del marco del empleo formal y en empresas legalmente constituidas, donde sus procesos contractuales estén en sentido estricto acogidos a la legislación laboral vigente en el país y en consonancia con los acuerdos internacionales en materia de empleo (Pacto de Productividad. Promoviendo la Inclusión Laboral de las personas con discapacidad, 2015).

A nivel internacional, en diferentes estudios, se ha indicado que una de las principales barreras que presenta las personas con discapacidad para lograr una verdadera inclusión social, es el acceso al empleo (Alcover, Rodríguez, Pastor, Fernández, \& Chambel, 2017; Shwazer, 2016; Zondek, A, 2015). Esta situación se confirma con las cifras reportadas por la OIT; ya que indica que en el mundo de 1.000.000.000 personas con discapacidad por lo menos 785.000.000 están en edad de trabajar, pero se enfrentan a múltiples dificultades para ingresar a un empleo decente, y a barreras actitudinales, físicas y de información que dificultan el disfrute a la igualdad de oportunidades en el mundo laboral (Organización Internacional del Trabajo, 2015).

Igualmente, Organismos multilaterales como la Organización Mundial de la Salud (OMS) y el Banco Mundial (BM), se refieren a diversas barreras que presentan las personas con discapacidad en el mundo laboral tales como la discriminación, prejuicios o falta de incentivos en los sistemas. Situaciones que se presentan a pesar de que en muchos países existen leyes que prohíben la discriminación en razón a la discapacidad a fin de mejorar el acceso a la economía formal, servicios relacionados con el empleo y capacitación laboral (Organización Mundial de la Salud \& Banco Mundial, 2011). 
Por su parte, autores como Montero prefieren utilizar el término desventaja laboral en vez de barreras e indican que se refiere a la situación que enfrenta una persona con discapacidad en comparación con otras personas; debido no solo a sus características asociadas a la discapacidad, sino más bien a las dificultades que haya presentado en el acceso a las oportunidades educacionales, de formación profesional, de movilidad en el entorno y otros aspectos como la discriminación (Montero, 2013).

En esta misma línea, otros autores señalan que las barreras enfrentadas por las personas con discapacidad en materia de empleo se deben al tema de la discriminación - perjuicios, estigmas y barreras - que impacta de manera directa y negativa; en otros determinantes sociales se connota en tema económico, de educación, movilidad, acceso a vivienda, y en si mimos a la vulneración de la dignidad humana; lo anterior por la falta de una debida implementación de las normas, ya que no hay una articulación entre las entidades del Estado, obstáculos socioeconómicos, físicos y legales donde lo que se debería trabajar es más un cambio de actitud combatiendo la discriminación y emprendiendo acciones para la inserción laboral que contrarresten dichos paradigmas. (Rodríguez \& Rico, 2009)

Concretamente en América Latina y el Caribe, se afirma que entre el $80 \%$ y el $90 \%$ de las personas con discapacidad están desempleadas y casi todos aquellos que tienen trabajo reciben salarios muy bajos o incluso ninguna compensación monetaria (Cecchini \& Martínez C., 2011). En el caso de Colombia, solamente se encuentran trabajando aproximadamente el 5,01\% personas con discapacidad (Ministerio de Salud y Protección Social, 2018).

En el caso específico de Colombia, la Ley 1618 para garantizar los derechos de las personas con discapacidad, en su artículo 2, núm. 5, señala que una barrera es "cualquier tipo de obstáculo que impida el ejercicio efectivo de los derechos de las personas con algún tipo de discapacidad" (Congreso de la República de Colombia, 2013a), las cuales a su vez se encuentran asociadas al mundo del trabajo. En este sentido se presentan de la siguiente forma. Figura 1.

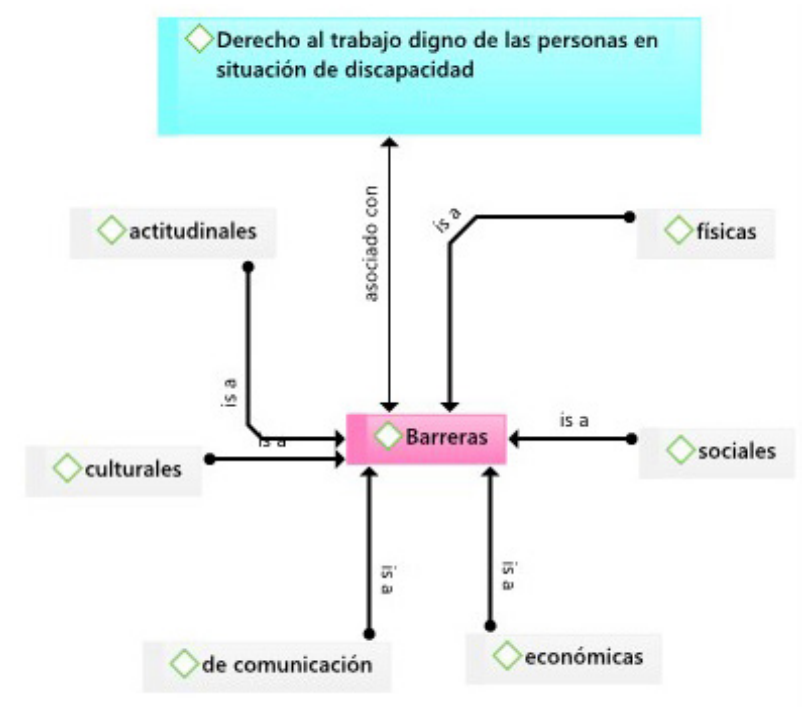

Figura 1. Barreras relacionadas con el derecho al trabajo digno de las personas con discapacidad

Fuente: construcción propia a partir de la Ley 1618 de 2013 
De forma particular, en el referente normativo que encontramos en la Ley 1618 solamente se explica y/o describe las barreras actitudinales, comunicativas y físicas:

"Actitudinales: Aquellas conductas, palabras, frases, sentimientos, preconcepciones, estigmas, que impiden u obstaculizan el acceso en condiciones de igualdad de las personas con y/o en situación de discapacidad a los espacios, objetos, servicios y en general a las posibilidades que ofrece la sociedad.

Comunicativas: Aquellos obstáculos que impiden o dificultan el acceso a la información, a la consulta, al conocimiento y en general, el desarrollo en condiciones de igualdad del proceso comunicativo de las personas con discapacidad a través de cualquier medio o modo de comunicación, incluidas las dificultades en la interacción comunicativa de las personas.

Físicas: Aquellos obstáculos materiales, tangibles o construidos que impiden o dificultan el acceso y el uso de espacios, objetos y servicios de carácter público y privado, en condiciones de igualdad por parte de las personas con discapacidad" (Congreso de la República de Colombia, 2013a).

Por otro lado, las capacidades actitudinales y comunicativas, también han sido definidas como "las consecuencias observables de las costumbres, practicas, ideologías, valores, normas, creencias reales y creencias religiosas que influyen en el comportamiento y la vida social de las personas en todos los ámbitos, desde las relaciones interpersonales y las asociaciones comunitarias hasta las estructuras políticas, económicas y legales" (Pacto de Productividad. Promoviendo la Inclusión Laboral de las personas con discapacidad, 2015); y se estima que uno de los principales obstáculos en materia de inclusión laboral por imaginarios o supuestos que se tienen respecto a sus condiciones de salud y capacidades para relacionarse en la sociedad (Centros para el control y la prevención de las enfermedades, 2019; Flores, Jenaro, Tomșa, López, \& Beltrán, 2014; Puin, 2002). En concordancia a estas barreras, se ha afirmado que aquellas experimentadas con mayor frecuencia por las personas que tienen discapacidades son las que afectan la audición, el habla, la lectura, la escritura o el entendimiento; por lo cual, usan maneras de comunicarse diferentes a las empleadas por quienes no tienen dichas discapacidades (Centros para el control y la prevención de las enfermedades, 2019).

Una reflexión que pude surgir de las barreras actitudinales y comunicativas es la importancia que tiene el papel del empleador al momento de realizar la inclusión laboral, a partir de estrategias tendientes a una mayor comprensión de la diversidad del ser humano y en esta medida lograr que en los ambientes de trabajo se comprenda la discapacidad más allá de las deficiencias físicas, psíquicas y sensoriales que puede presentar una persona en cualquier momento de la vida.

Sobre las barreras físicas, se indica que no hay diseño e implementación de calles, avenidas, edificios, sistema de transporte e infraestructura accesible tanto privadas como públicas que permitan poner al mismo nivel a la persona con discapacidad (Centros para el control y la prevención de las enfermedades, 2019). En Colombia es un problema que se empezó a reconocer desde la Ley 361 (Congreso de la República de Colombia, 1997, p. 361) y sin lugar a dudas, esta barrera requiere un compromiso del Estado para lograr dos propósitos principales de la Convención de los Derechos de las Personas con Discapacidad, el acceso universal y el diseño para todos. 
El primero, acceso universal, entendido como como "la elaboración de productos, entornos, programas y servicios que puedan utilizar todas las personas, en la mayor medida posible, sin necesidad de adaptación ni diseño especializado"; y el segundo, diseño para todos, como "la necesidad de asegurar el acceso de las personas con discapacidad en igualdad de condiciones con las demás, al entorno físico, el transporte, la información y las comunicaciones, incluidos los sistemas y las tecnologías de la información y las comunicaciones, y a otros servicios e instalaciones abiertos al público o de uso público, tanto en zonas urbanas como rurales" (Organización de Naciones Unidas, 2006). En este sentido, si bien es importante el compromiso estatal, en materia de empleo se pueden realizar avances como una política tanto de entidades públicas como privadas en la construcción y adecuación de espacios de inclusión general.

Con respecto a las barreras, sociales y culturales, el Centro para el control y la prevención de la enfermedad reúne elementos de interés al definirlas de la siguiente manera (Centros para el control y la prevención de las enfermedades, 2019):

"Sociales: tienen que ver con las condiciones en que las personas nacen, crecen, viven, aprenden, trabajan y envejecen o con los determinantes sociales de la salud, que pueden contribuir a reducir el funcionamiento entre las personas con discapacidades

Culturales: se mencionan donde el Estado, la sociedad en general y las empresas han jugado un rol bastante importante viendo a la persona en situación de discapacidad como una tragedia personal y familiar restringiendo sus oportunidades en mercado laboral".

En cuanto a las culturales y sociales, planteamientos como los de Flores et al sobre la sobrecarga que pueden verse sometidas y falta de autonomía en el campo laboral, evidencian la necesidad de un cambio social y cultural; en sentido de reconocer que la discapacidad no es un asunto de la persona sino del reconocimiento de la diversidad de los seres humanos por parte de todos (Flores et al., 2014). Es así como en el campo laboral, el respecto por la dignidad de las personas con discapacidad debe darse en todos los niveles de la estructura organizacional.

Referente a las barreras económicas se ha señalado que están relacionadas con las dificultades para el acceso a la educación y a la inadecuada e insuficiente formación que se ofrece en muchas fundaciones de inserción laboral. Asimismo, se indica que tienen mayores dificultades para acceder a la salud y la posibilidad de realizar un proceso de rehabilitación integral (Rodríguez \& Rico, 2009). Lo anterior, puede generar que las posibilidades de empleo de algunas personas con discapacidad se vean reducidas en la medida que la oferta se limita, tanto en puestos de trabajo como posibilidad de ascensos, por el escaso nivel educativo y/o de preparación.

En consideración a esta desventaja laboral y/o barreras, que pueden presentar las personas con discapacidad, se considera que la inclusión laboral cumple un rol determinante en el marco del trabajo decente y donde este proceso se sustenta "en la igualdad, la equiparación de oportunidades y la plena participación social, y, en la práctica, se concreta una vez la persona se incorpora a un trabajo y participa de todas las actividades laborales y sociales de la empresa" (Montero, 2013). En dicha medida es necesario trascender el discurso a prácticas que contribuyan a una efectiva inclusión social como se explica a continuación. 


\section{Algunas alternativas para minimizar las barreras de acceso al trabajo digno de las personas con discapacidad}

Ante el reconocimiento internacional de barreras en el campo laboral, la OIT ha indicado que actualmente es necesario aplicar un doble enfoque para la inclusión de las personas con discapacidad. El primero, referido a los programas o iniciativas específicas destinadas a superar las desventajas o barreras particulares; y el segundo, orientado a la inclusión en los servicios y actividades de carácter general como la formación profesional, la promoción del empleo, planes de protección social y estrategias para la reducción de la pobreza (Organización Internacional del Trabajo, 2015).

Desde esta óptica el compromiso de los países miembros de esta organización implica que, adicionalmente al reconocimiento de las barreras para la participación y la igualdad de oportunidades, se tracen medidas para el cumplimiento de los preceptos legales; lo cual supone un trabajo colaborativo ante la imposibilidad de atender de forma individual o institucional las necesidades de las personas con discapacidad. De esta manera, es necesaria la intervención de organizaciones internacionales, instituciones estatales, organizaciones privadas y empresas para lograr concretamente, en materia de empleo, el acceso al trabajo digno.

En el marco de las organizaciones internacionales, además de la OIT, sobresale la labor realizada por el Banco Mundial (BM) y la Organización Iberoamericana de la Seguridad Social (OISS). El Banco Mundial, a través de la promoción del fin de la pobreza extrema para lograr la inclusión de las personas con discapacidad, los estudios analíticos, las políticas sobre buenas prácticas de empleo y elaboración de documentos sobre medidas razonables para adecuar los lugares de trabajo. Asimismo, para el BM, en concordancia con los objetivos del Desarrollo Sostenible (ODS), son esferas importantes la educación, el empleo y el trabajo decente de las personas con discapacidad (Banco Mundial, 2019).

Por su parte, la OISS, dentro de sus objetivos, plantea fomentar el intercambio de buenas prácticas entre gobiernos de Iberoamérica acerca de las legislaciones y las políticas activas de inserción laboral de personas con discapacidad. Un producto significativo de esta iniciativa ha sido el estudio de las medidas para la promoción del empleo de las personas con discapacidad. (Organización Iberoamericana de Seguridad Social, 2014) y aunque se realiza por cada país, es decir, no se hace un análisis comparado como tal, si permite evidenciar el desarrollo normativo, especialmente en materia de empleo y seguridad social de los Estados miembros, además de las estrategias adicionales que se realizan para lograr un empleo digno de las personas con discapacidad.

En el estudio de la OISS respecto a Colombia, se destaca que las estrategias que minimizan las barreras están apalancadas por la normatividad; resalta especialmente la protección en caso de despidos conforme a la Ley 361 de 1997, la inclusión de las personas con discapacidad en la regulación del teletrabajo y los beneficios tributarios para las empresas comprometidas con la vinculación laboral de personas con discapacidad (Organización Iberoamericana de Seguridad Social, 2014). No obstante, la protección establecida de la citada Ley 361 ha generado una interpretación jurisprudencial de la Corte Constitucional, tendiente no solo a proteger a las personas con discapacidad sino a quienes por cualquier razón estén afectadas en su estado de salud y le impida desarrollar su actividad laboral (Corte 
Constitucional, 2017, 2019), presupuesto que para diferentes sectores, se ha convertido en un obstáculo para la contratación de personas con discapacidad ante el temor de los empleadores de no poder terminar la relación laboral sin que medie la estabilidad ocupacional reforzada, lo cual en últimas se convierte en un asunto discriminatorio.

En cuanto a instituciones estatales, la Ley 1636 reconoce como agencia de gestión y colocación de empleo al Servicio Nacional de Aprendizaje (SENA) con apoyo de las Cajas de Compensación (Congreso de la República de Colombia, 2013b). Respecto al SENA, se ha destacado el papel que cumple el observatorio laboral para la selección de programas de formación pertinentes con la dinámica del mercado laboral y la asesoría y acompañamiento a las empresas (Mahecha, Pacto de Productividad-Equipo Técnico, \& Fundación Corona-Equipo Técnico, 2018). Concretamente se ha indicado que a través del programa SENA Incluyente, se han adelantado acciones de formación profesional en los 115 Centros de Formación del país y otorgado certificaciones de competencias laborales e intermediación laboral a través de los Centros del Sistema Nacional de Recurso Humano (SNRH); haciendo énfasis en la inclusión del colectivo de personas con discapacidad en los ambientes de aprendizaje, dependiendo de las capacidades y potencialidades que tienen las personas con discapacidad sensorial, física, cognitiva y mental (Ministerio de Salud y Protección Social, 2017).

Adicionalmente, la Unidad Administrativa Especial del Servicio Público de Empleo (UASPE) del Ministerio de trabajo creada a través de la Ley 1636 de 2013, incluyó a las personas con discapacidad dentro del programa Mitigación de Barreras; el cual cuenta con un Fondo de Oportunidades de Empleo (FOE) para que a través de centros de empleo, se facilite el encuentro entre la oferta y la demanda laboral de las poblaciones de difícil inserción laboral (Ministerio de Trabajo, 2019).

Por otro lado, el gobierno nacional ha implementado un portal de teletrabajo que tiene entre una de sus líneas la inclusión social de personas con discapacidad. Por ejemplo, se plantea el tema de inserción de las personas con discapacidad física, el papel del empleador y la posibilidad hoy de vincularse bajo esta modalidad teniendo en cuenta que es posible trabajar a diario, demostrar resultados y cumplir metas sin salir de casa (Ministerio de Tecnologías de la Información y las Comunicaciones, 2019).

También debe destacarse que, en materia de fuentes de información, en Colombia se ha avanzado y se cuenta con el Registro para la Localización y Caracterización de las Personas con Discapacidad (RLCPD) como única fuente de datos oficial y válida para identificar a las personas con discapacidad en Colombia, a cargo del Ministerio de Salud y Protección Social, según lo establecido en el literal e) del artículo 10 de la Ley Estatutaria 1618 de 2013. Esta herramienta ha sido muy útil para identificar las personas con discapacidad activas en el mercado laboral, los rangos de edad, nivel de educación y sector formal o informal en el que desarrollan actividades (Ministerio de Salud y Protección Social, 2019).

De igual modo, en Colombia, como una iniciativa ejecutada de manera interinstitucional por Fundación Corona, Banco Interamericano de Desarrollo (BID), la Fundación Saldarriaga Concha, la Agencia Presidencial de Cooperación Internacional de Colombia (APC), el Servicio Nacional de Aprendizaje (SENA), el Ministerio del Trabajo y cuatro Cajas de Compensación Familiar: Cafam en la ciudad de Bogotá, 
Comfenalco Antioquia en Medellín, Comfandi en Cali y Comfamiliar Risaralda; se creó el programa Pacto de Productividad, que tiene como objetivo ayudar a las empresas a estructurar procesos de incorporación laboral de personas en situación de discapacidad, favoreciendo tanto a las empresas como a las personas con discapacidad a través de un modelo que reconoce y promueve el derecho a vincularse laboralmente como todos los demás, materializa las recomendaciones hechas por Naciones Unidas y los firmantes de la Convención de los Derechos de las Personas con Discapacidad, facilitando una hoja de ruta para que los involucrados identifiquen sus roles y acciones en materia de inclusión. (Pacto de Productividad. Promoviendo la Inclusión Laboral de las personas con discapacidad, 2015).

Entre los logros del "Pacto de Productividad" se cuenta en el componente de la Movilización del sector empresarial con la asesoría en los procesos de diagnóstico de oportunidades y barreras, análisis de puestos de trabajo, elaboración de un plan de ajustes, acompañamiento en los procesos de selección y talleres de capacitación; además de seguimiento por 6 meses una vez se ha logrado la ubicación laboral (Pacto de Productividad, 2019).

Como resultado, en el componente de fortalecimiento de las instituciones de inclusión laboral se ha logrado vinculaciones como aprendices del SENA y la realización de programas de formación y en el elemento de articulación y difusión del programa, se han realizado visitas a instituciones de y para personas con discapacidad con el propósito de construir un mapa de servicios y entidades que participan de manera directa o indirecta en procesos de inclusión laboral (Pacto de Productividad, 2019).

Aunque el programa mencionado muestra un avance significativo en la implementación de estrategias en el ámbito laboral para las personas con discapacidad en el país, el único estudio publicado sobre las barreras para la inclusión por parte de Pacto de Productividad (Programa Pacto de Productividad, 2010) refleja lo siguiente: baja participación en los cargos directivos, ya que los puestos que se ofrecen son para actividades asistenciales; ausencia del tema de desarrollo de capacidades, fue planteado desde la Política Nacional de Discapacidad como una meta de la inclusión y en el estudio se omite esta perspectiva de análisis para la empleabilidad, las cifras de personas empleadas no son significativas respecto a las estadísticas nacionales de personas con discapacidad en edad productiva; por lo tanto, es solamente un referente teniendo en cuenta que no hay cifras oficiales de desempleo de las personas con discapacidad en Colombia y no se plantean acciones para contextualizar las políticas y estrategias implementadas dentro de las empresas con las condiciones de salud, empleo y trabajo de esta población.

Con relación a programas de carácter privado, se puede resaltar los emprendidos por la Fundación Saldarriaga Concha, Grupo Social Empresarial Arcángeles, Best Buddies Colombia y la empresa Accenture Technology, entre otras, con la finalidad de promover el empleo de las personas con discapacidad en Colombia como se explica a continuación:

La Fundación Saldarriaga Concha es una entidad sin ánimo de lucro comprometida con las personas con discapacidad y los adultos mayores. Su cadena de valor está definida en las etapas que deben cumplir todos los proyectos que realiza la fundación; estas son entender, aprender y materializar con lo que se pretende que los proyectos estén acompañados de acciones permanentes de visibilización, incidencia, gestión del conocimiento y monitoreo. Relativo al campo laboral de las personas 
con discapacidad, se destaca su incidencia en las políticas públicas, el desarrollo de documentos sobre los temas coyunturales que el país debe trabajar para avanzar en la inclusión social y el relacionamiento con las empresas («Fundación Saldarriaga Concha», 2019).

Esta fundación participa como aliado en el citado programa estatal Mitigación de Barreras, concretamente orienta el proceso de formulación, selección de proyectos y realiza seguimiento a su implementación. Además, soporta los procesos administrativos que dan viabilidad a la ejecución de los proyectos y, por último, asigna los recursos financieros con base en las necesidades particulares de los mismos (Ministerio de Trabajo, Unidad del Servicio de Empleo, \& Fundación Saldarriaga Concha, 2017)

Igualmente, el Best Buddies Colombia es una comunidad apoyada internacionalmente, que promueve la empleabilidad de la población con discapacidad intelectual en Colombia, y también trabaja en pro de la inclusión bajo tres lineamientos: el desarrollo de habilidades sociales a través de la amistad, la generación de oportunidades laborales y la consecución de recursos que permitan ampliar el impacto positivo sobre las personas con discapacidad en Colombia; el programa está en 36 ciudades del país y tiene vinculadas más de 600 personas trabajando en más de 70 empresas tales como Alkosto, Terpel, Claro, Doria, Bavaria, el Espectador entre otros (Best Buddies Colombia, 2019).

Se ha resaltado el compromiso en la formación de personas con discapacidad de Best Buddies Colombia por medio de la alianza con el SENA y las empresas privadas en programas como Técnico en Desarrollo de Operaciones Logísticas en la Cadena de Abastecimiento, Auxiliar Logístico en Almacenes de Cadena y Auxiliar Logístico en Eventos y Servicios(Best Buddies Colombia, 2019).

Así mismo se destaca un proyecto con el Banco Interamericano de Desarrollo 20182020 que tiene como principal objetivo el desarrollo de un modelo de inclusión productiva que brinde capacitación en habilidades socioemocionales, adaptativas y servicios de intermediación laboral para expandir las oportunidades de empleo y promover emprendimientos de Personas con Discapacidad (Best Buddies Colombia, 2019).

Por otra parte, la Fundación Arcángeles, es una entidad sin ánimo que surgió para aportar a la rehabilitación integral de personas con discapacidad. Actualmente es un proyecto conjunto de cinco empresas que trabajan por la inclusión de poblaciones vulnerables, a través de un movimiento activista, desarrollando su gestión con dos enfoques: la salud y el desarrollo de programas y proyectos. En el campo laboral tiene un programa de inserción que cumple el objetivo de generar espacios propicios para que la población vulnerable y en especial las personas con discapacidad y contempla acciones dirigidos a las empresas para sensibilizar y asesorar sus procesos de talento humano para garantizar que estos cuenten con enfoque en diversidad. También evalúa, capacita y empodera a la población partiendo de sus propias competencias personales y laborales, para que accedan al mundo del trabajo de manera competitiva y equitativa con el fin de mitigar la violación de sus derechos y ejercer el goce efectivo de los mismos (Fundación Arcángeles, 2019).

En el mercado laboral, la estrategia de inclusión de Arcángeles con mayor reconocimiento ha sido el modelo de vigilancia inclusiva al iniciar laborales en el año 2016 
y aplicada inicialmente en el Centro Comercial Gran Estación en Bogotá con la empresa Securitas. Esta iniciativa la han replicado en otros países como Chile, Argentina, Uruguay, Perú y Costa Rica con el apoyo de esta Fundación (Fundación Arcángeles, 2019).

En cuanto a la empresa Acceture Technology, dedicada a la interacción entre el negocio y la tecnología, se encuentra que tiene presencia en Colombia desde el año 2006 con un programa denominado "sin barreras" para facilitar las oportunidades de empleo de las personas con discapacidad, brindándoles el apoyo y el seguimiento necesario para la adecuada adaptación al mundo laboral a través de capacitación, reconocimientos, crecimiento, motivación (Acceture Technology, 2019). Sin embargo, se trata de un programa limitado a un grupo específico de la población con discapacidad, pues los perfiles de búsqueda y empleabilidad se enfocan a estudiantes o profesionales graduados de carreras como sistemas e ingeniería, ciencias sociales y ciencias económicas.

De acuerdo con lo anterior, los ejemplos enunciados de estrategias para minimizar las barreras de acceso al trabajo digno de las personas con discapacidad son importantes en el campo de inclusión social y, en una u otra medida, han contribuido a la empleabilidad, así como a un cambio de visión respecto a la diversidad de capacidades de todos los seres humano y la posibilidad de participar en el mercado laboral. También es relevante que algunas de las propuestas como Best Buddies y Fundación Arcángeles surjan de personas con discapacidad y/o sus familias, lo que demuestra un mayor empoderamiento de un cambio en la percepción de la discapacidad como un problema que atañe a la sociedad por las barreras para la participación y la igualdad a la que se han visto expuesta en el transcurso de la historia.

Por el contrario, las cifras reflejadas tanto a nivel nacional e internacional sobre desventajas laborales y desempleo de las personas con discapacidad respecto a las demás, permite plantear el cuestionamiento que estas estrategias se tornan insuficientes en materia laboral; de los ejemplos planteados, en la práctica, se encuentran criticas como la concentración en discapacidades especificas dentro de las empresas que finalmente resultan siendo solamente incluyentes para subgrupos de la población y por esto las capacidades en condiciones de igualdad respecto a otros trabajadores quedan en un segundo plano limitándose la oferta a los campos específicos. En razón a los campos específicos, además de las limitaciones del público al que van dirigido, los perfiles ocupacionales se orientan más a labores misionales; por lo que, posiblemente, pueden restringirse oportunidades para personas con discapacidad que sean profesionales en distintos campos del conocimiento. En dicho sentido, es crear puentes más sólidos de carácter educativo y empresarial que no solamente permitan el acceso al empleo sino condiciones de permanencia y seguimiento en materia laboral a las personas con discapacidad.

\section{Conclusiones}

Los referentes legales en Colombia son un avance importante para el acceso al empleo y la concientización de las empresas para implementar una política inclusiva dentro de sus organizaciones y se encuentran acordes con la Convención de los Derechos de las Personas con Discapacidad. Sin embargo, sigue ausente un marco regulador de las iniciativas legales de accesibilidad laboral, especialmente de lo indicado en la Ley 1618 de 2013. Se requiere ir más allá de un compromiso social y plantear obligaciones claras para las empresas, así como garantías a los 
empleadores y trabajadores que participen activamente en la minimización de barreras para la participación y la igualdad de condiciones.

Sobre las barreras para el trabajo digno de las personas con discapacidad, las estadísticas mundiales y nacionales reflejan las desventajas que presentan en relación a las personas sin discapacidad en el campo laboral. En el país, la Ley Estatutaria 1618 de 2013 reconoce la existencia de estas; describe las actitudinales, las comunicativas y las físicas; y enuncia otras como las sociales, las culturales y las económicas. Todas esas barreras han sido estudiadas por diferentes autores para referirse a la importancia de la equiparación de oportunidades y la plena participación social, la cual se logra a través de estrategias o programas por parte del Estado y/o particulares.

Sobre estrategias, en un análisis global, se encuentra que organismos multilaterales como la OIT, BM, BID y OISS han participado en propuestas para lograr la inclusión de personas con discapacidad en Colombia y como resultado surgen medidas y programas que contribuyen al trabajo decente de esta población como por ejemplo Pacto de Productividad. A su vez, en Estado Colombiano, se ha asumido un papel protagónico, especialmente a través del SENA y las Cajas de Compensación con la finalidad de consolidarse no solo como bolsas de empleo sino de formación para el trabajo de las personas con discapacidad. Igualmente, existen instituciones de carácter privado que han contribuido al derecho al trabajo digno de las personas con discapacidad como la Fundación Saldarriaga Concha, Best Buddies Colombia, la Fundación Arcángeles y la empresa Acceture Technology, que aportan de una u otra forma a minimizar las barreras para la participación y la igualdad.

No obstante, el panorama nacional e internacional refleja que aún persisten las barreras y que las estrategias planteadas tanto estatales como privadas no cumplen las expectativas, entre otras razones, porque son incluyentes solo para algunos subgrupos de las personas con discapacidad, los campos laborales son reducidos y los perfiles ocupacionales se orientan más a labores misionales., al contrario, no puede negarse que representan un avance importante en materia de inclusión laboral para esta población.

Finalmente, es necesario crear puentes más sólidos de carácter educativo y empresarial que no solamente permitan el acceso al empleo sino también condiciones de permanencia y seguimiento en materia laboral a las personas con discapacidad. En el caso de Colombia los retos no son normativos sino de aplicación de los referentes legales y jurisprudenciales; lo cual esto involucra además de los empleadores, a los trabajadores; quienes también deben ser conscientes que desde la perspectiva de la discapacidad, las barreras para la participación y la igualdad que se presenta por una deficiencia física, síquica o sensorial, se pueden presentar en cualquier etapa de la vida y las mismas no pueden ser un obstáculo para desarrollar capacidades en el campo laboral sea por la ausencia o extralimitación de la estabilidad ocupacional reforzada, la poca oferta laboral, las restricciones de acceso a la educación y/o la estigmatización social. 


\section{Referencias}

Acceture Technology. (2019). Oportunidades de Carrera en Accenture | Colombia. Recuperado 15 de agosto de 2019, de Acceture Technology website: https://www. accenture.com/co-es/careers

Alcaldía de Medellín. Política Pública en Discapacidad para el municipio de Medellín. Pub. L. No. 2009, Acuerdo 8618 (2009).

Asamblea Nacional Constituyente. Constitución Política de Colombia. , (1991).

Banco Mundial. (2019). Discapacidad [Text/HTML]. Recuperado 2 de agosto de 2019, de World Bank website: https://www.bancomundial.org/es/topic/disability

Benach, J., \& Muntaner, C. (Eds.). (2010). Empleo, trabajo y desigualdades en salud: Una visión global (1. ed). Barcelona: Icaria Editorial.

Benavídez, F., Boix, P., Rodrigo, F., \& Gil, J. (2013). Informes de Salud Laboral. España 2001 2010 (p. 95). Recuperado de CISAL-UPF website: http://www.aespla.com/attachments/article/57/2013 02 ISL 2001-2010.pdf

Best Buddies Colombia. (2019). Best Buddies Colombia. Recuperado 22 de abril de 2019, de .: Best Buddies Colombia-Amigos del Alma :. website: https://www. bestbuddies.org.co

Cecchini, S., \& Martínez C., R. (2011). Protección social inclusiva en América Latina: Una mirada integral, un enfoque de derechos (1. ed). Santiago de Chile: Naciones Unidas, Comisión Económica para América Latina y el Caribe.

Centros para el control y la prevención de las enfermedades. (2019, abril 25). Las discapacidades y la salud. Recuperado 4 de agosto de 2019, de https://www.cdc.gov/ ncbddd/spanish/disabilityandhealth/disability-barriers.html

Congreso de la República de. Ley 1429. , Pub. L. No. Diario Oficial No. 47.937 de 29 de diciembre de 2010 (2010).

Congreso de la República de Colombia. Ley 361. , Pub. L. No. Diario Oficial 42.978 del 11 de febrero (1997).

Congreso de la República de Colombia. Ley 789. ,Pub. L. No. Diario Oficial No 45.046 de 27 de diciembre de 2002 (2002).

Congreso de la República de Colombia. Ley 1618. , Pub. L. No. Diario Oficial 48.717 de 27 de febrero de 2013 (2013).

Congreso de la República de Colombia. Ley 1636. , Pub. L. No. Diario Oficial 4.8825 (2013).

Consejo Nacional de Política Económica y Social. (2013). Conpes Social 166. Recuperado de http://repository.javeriana.edu.co/handle/10554/15548

Corte Constitucional. C-425. , (2005).

Corte Constitucional. SU-049., (2017).

Corte Constitucional. SU-040., (2018).

Corte Constitucional. C-200. , (2019).

Discapacidad y trabajo [Documento]. (2016, abril 28). Recuperado 16 de agosto de 2016, de http://www.ilo.org/global/topics/disability-and-work/WCMS 475652/ lang--es/index.htm

Farné, S. (2003). Estudio sobre la calidad del empleo en Colombia (2a ed.; Organización Internacional del Trabajo, Ed.). Recuperado de http://datateca.unad.edu.co/contenidos/111001/2015 2/UNIDAD UNO/LEGISLACION LABORAL EN COLOMBIA.pdf

Flores, N., Jenaro, C., Tomșa, R., López, J., \& Beltrán, M. (2014). Actitudes, barreras y oportunidades para el empleo de personas con discapacidad intelectual. Revista de Derecho. Comunicaciones y Nuevas Tecnologías, 15, 1-24.

Fundación Arcángeles. (2019). Programas y Proyectos. Recuperado 15 de agosto de 2019, de Arcángeles website: https://arcangeles.org/programas-y-proyectos/ 
Fundación Saldarriaga Concha. (2019). Recuperado 15 de junio de 2015, de Fundación Saldarriaga Concha website: http://www.saldarriagaconcha.org/como-lo-hacemos/modelo-de-intervencion

García, A. M., Delclós, J., \& Benavides, Fernando. (2013). Trabajo y salud. En Salud laboral: Conceptos y técnicas para la prevención de riesgos laborales (4a ed., pp. 17-25). Barcelona: Elsevier.

International Labour Organization (Ed.). (2014). World Social Protection Report. Building economic recovery, inclusive development and social justice (1 a ed.). Recuperado de http://www.ilo.org/wcmsp5/groups/public/---dgreports/---dcomm/documents/ publication/wcms 245201.pdf

Mahecha, P., Pacto de Productividad-Equipo Técnico, \& Fundación Corona-Equipo Técnico. (2018). Los servicios de formación para el trabajo como parte del modelo de inclusión laboral de personas con discapacidad «Pacto de Productividad». Recuperado de Pacto de productividad website: https://agenciapublicadeempleo.sena.edu. co/Documents/MODELO\%20DE\%20INCLUSI\%C3\%93N\%20LABORAL\%20DE\%20 PERSONAS\%20CON\%20DISCAPACIDAD.pdf

Metts, R. L. (2000). Disability issues, trends, and recommendations for the World Bank (1a ed.). Recuperado de http://siteresources.worldbank.org/DISABILITY/Resources/280658-1172606907476/DisabilitylssuesMetts.pdf

Ministerio de Hacienda y Crédito Público. Decreto 392. , Pub. L. No. Diario Oficial 50.519 (2018).

Ministerio de Salud y Protección Social. (2015). Sala Situacional de Personas con Discapacidad (p. 56). Recuperado de Ministerio de Salud y Protección Social website: https://www.minsalud.gov.co/sites/rid/Lists/BibliotecaDigital/RIDE/DE/PS/Sala-situacional-discapacidad-Nacional-agosto-2015.pdf

Ministerio de Salud y Protección Social. (2017). Abecé de la discapacidad. Recuperado de https://www.minsalud.gov.co/sites/rid/Lists/BibliotecaDigital/RIDE/DE/PS/ abece-de-la-discapacidad.pdf

Ministerio de Salud y Protección Social. (2018). Sala situacional de las Personas con discapacidad. Recuperado 13 de enero de 2019, de http://ondiscapacidad.minsalud.gov.co/Documentos\%20compartidos/Sala\%20situaci\%c3\%b3n\%20Discapacidad\%20septiembre\%202018.pdf

Ministerio de Salud y Protección Social. (2019). Registro para la Localización y Caracterización de Personas con Discapacidad. Recuperado de https://www.minsalud. gov.co/proteccionsocial/Paginas/DisCapacidad RLCPD.aspx

Ministerio de Tecnologías de la Información y las Comunicaciones. (2019). Inclusión social gracias al Teletrabajo. Recuperado 14 de agosto de 2019, de Portal Teletrabajo Colombia website: https://www.teletrabajo.gov.co/622/w3-article-14277. $\underline{\text { html }}$

Ministerio de Trabajo. Decreto 2011. , Pub. L. No. Diario Oficial No. 50125 del 23 de enero de 2017 (2017).

Ministerio de Trabajo. Decreto 2177. , Pub. L. No. Diario Oficila No 50455 del 22 de diciembre de 2017 (2017).

Ministerio de Trabajo. (2019). Funciones y Deberes I Unidad del Servicio Público de Empleo. Recuperado 14 de agosto de 2019, de Unidad del Servicio Público de Empleo website: https://unidad.serviciodeempleo.gov.co/la-unidad/funciones-y-deberes/

Ministerio de Trabajo, Unidad del Servicio de Empleo, \& Fundación Saldarriaga Concha. (2017). Programa de mitigación de barreras para poblaciones de dificil inserción laboral. Recuperado de https://www.saldarriagaconcha.org/wp-content/uploads/2019/01/programa empleo Final ISBN.pdf 
Montero, C. (2013). Estrategias para facilitar la inserción laboral a personas con discapacidad (1 a ed.). Costa Rica: Editorial Universidad Estatal A Distancia.

Moreno, M., Cortés, E., Cárdenas, A., Giraldo, Z., \& Mena, L. (2013). Valoración ocupacional: Un desplazamiento conceptual basado en la capacidad de las personas con discapacidad. Revista de Salud Pública, 15(5), 764-777.

Organización de Estados Americanos. Convención interamericana para la eliminación de todas las formas de discriminación contra las personas con discapacidad. , (1999).

Organización de Naciones Unidas. Convención sobre los derechos de las personas con discapacidad. , (2006).

Organización Iberoamericana de Seguridad Social. (2014). Medidas para la promoción del empleo de las personas con discapacidad en Iberoamérica (1 a ed.). Recuperado de http://www.oiss.org/lMG/pdf/Oiss Estudio sobre medidas promocion de empleo.pdf

Organización Internacional del Trabajo. Convenio 159. , (1983).

Organización Internacional del Trabajo. Normas Uniformes sobre la igualdad de oportunidades para las personas con discapacidad. , Pub. L. No. Resolución No 48 (1996).

Organización Internacional del Trabajo. (2004). ¿Qué es el trabajo decente? [Noticia]. Recuperado 15 de agosto de 2016, de http://www.ilo.org/americas/sala-de-prensa/WCMS LIM 653 SP/lang--es/index.htm

Organización Internacional del Trabajo. (2015). Estrategia y plan de acción para la inclusión de la discapacidad 2014-17: Un doble enfoque de acciones transversales y específicas para las personas con discapacidad (1a ed.). Recuperado de http://www. ilo.org/wcmsp5/groups/public/---ed emp/---ifp skills/documents/genericdocument/wcms 370773.pdf

Organización Mundial de la Salud, \& Banco Mundial. (2011). Informe mundial sobre discapacidad (p. 388). Recuperado de OMS website: http://www.who.int/disabilities/ world report/2011/es/

Pacto de Productividad. (2019). Reporte de logros del programa pacto de productividad. Recuperado de https://bit.ly/2UsNARz

Pacto de Productividad. Promoviendo la Inclusión Laboral de las personas con discapacidad. (2015). Recuperado de http://www.fundacioncoronamedios.org/pactodeproductividad2015/files/assets/common/downloads/publication.pdf

Palacios, A. (2008). Medidas relacionadas con la igualdad y la no discriminación en la implementación de la convención internacional sobre los derechos de las personas con discapacidad. 12. Recuperado de http://orff.uc3m.es/handle/10016/9900

Programa Pacto de Productividad. (2010). Estudio de barreras para la inclusión laboral de las personas con discapacidad (p. 58). Recuperado de Ministerio de Salud y Protección Social website: http://www.pactodeproductividad.com/pdf/estudiodebarrerasparalainclusionlaboraldepcd.pdf

Puin, D. C. (2002). Actitudes frente a la vinculación laboral de personas con discapacidad. Revista de la Facultad de Medicina, 50, 196-199.

Rodríguez, C., \& Rico, L. (2009). Discapacidad y derecho al trabajo. Recuperado de https:// derecho.uniandes.edu.co/es/publicaciones/colecciones/coleccion-estudios-cijus/1721-discapacidad-y-derecho-al-trabajo

Secretaría de Inclusión Social, Familia y Derechos Humanos. (2019). Unidad de Discapacidad-Ser Capaz. Recuperado 16 de abril de 2017, de Alcaldía de Medellín website: https://www.medellin.gov.co/irj/portal/medellin?NavigationTarget=navurl://0daf6d12049919fafad66823152c5f2d

Stang, M. F. (2011). Las personas con discapacidad en América Latina del reconocimiento jurídico a la desigualdad real (Población y Desarrollo N.o 103; p. 83). Recuperado de Centro Latinoamericano y Caribeño de Demografía (CELADE) website: http:// www.bivica.org/upload/discapacitados america latina.pdf 
Pág 24

Véliz, H. O., \& Espinoza, R. V. (2010). Empleo con apoyo: Un camino para la inserción laboral de las personas con discapacidad intelectual en Chile. UCMaule - Revista Académica de la Universidad Católica del Maule, (39), 52. (78403980). 